\title{
GRADIENT METHODS FOR SOLVING STOKES PROBLEM
}

\author{
I.I. GOLICHEV, T.R. SHARIPOV, N.I. LUCHNIKOVA
}

\begin{abstract}
In the present paper we consider gradient type iterative methods for solving the Stokes problem in bounded regions, where the pressure serves as the control; they are obtained by reducing the problem to that of a variational type. In the differential form the proposed methods are very close to the algorithms in the Uzawa family. We construct consistent finite-difference algorithms and we present their approbation on the sequence of grids for solving two-dimensional problem with a known analytic solution.
\end{abstract}

Keywords: Stokes problem, optimal control, gradient method, finite-difference scheme

Mathematics Subjects Classifications: 49M20, 35Q30, 93C05

\section{INTRODUCTION}

In a bounded domain $\Omega \subset \mathbb{R}^{n},(n=2,3)$ with a smooth boundary $S \in C^{2}$ (that is, twice continuously differentiable) we consider the Stokes problem

$$
\begin{aligned}
& -\nu \Delta \mathbf{v}=\mathbf{f}-\nabla p,\left.\mathbf{v}\right|_{S}=0, \\
& \operatorname{div} \mathbf{v}=0, \\
& (p, 1)_{L_{2}(\Omega)}=0,
\end{aligned}
$$

where $\mathbf{f}=\left(f^{(1)}, \ldots, f^{(n)}\right) \in \boldsymbol{L}_{2}(\Omega)=\left(L_{2}(\Omega)\right)^{n}, \mathbf{v}=\left(v^{(1)}, \ldots, v^{(n)}\right)$ is the velocity vector, $p$ is the pressure, $\nu>0$ is the coefficient of the kinematic viscosity.

In accordance with [1, Ch. III, Sect. 5, Thm. 2], under these assumptions, problem (1)-(3) has the unique strong solution $\mathbf{v} \in \boldsymbol{H}^{2}(\Omega)=\left(H^{2}(\Omega)\right)^{n}, \nabla p \in \boldsymbol{L}_{2}(\Omega)$. Hereinafter we employ standard notations for Sobolev spaces $H^{l}(\Omega) \equiv W_{2}^{l}(\Omega), l=1,2 \ldots$

We shall treat problem (1)-(3) as the inverse one for (1), (3), in which the pressure gradient $\nabla p$ is not known but an additional condition (2) is prescribed. Such problem can be formulated as an equivalent to the original problem of optimal control:

$$
J(\mathbf{u})=\frac{1}{2}\|\operatorname{div} \mathbf{v}(\mathbf{u})\|_{L_{2}(\Omega)}^{2} \rightarrow \inf , \mathbf{u} \in G(\Omega),
$$

where $G(\Omega)=\left\{\mathbf{u} \in \boldsymbol{L}_{2}(\Omega): \mathbf{u}=\nabla p, p \in H^{1}(\Omega),(p, 1)_{L_{2}(\Omega)}=0\right\}$ is the gradient component in the orthogonal expansion $\boldsymbol{L}_{2}(\Omega)$, while $\mathbf{v}(\mathbf{u})$ is a solution to the system

$$
-\nu \Delta \mathbf{v}(\mathbf{u})=\mathbf{f}-\mathbf{u},\left.\mathbf{v}(\mathbf{u})\right|_{S}=0 .
$$

The fact that many problems of the mathematical physics can be reduced to extremal problem is well-known and is widely used. Among known methods of solving stationary Stokes problem in primitive variables based on variational methods, we first of all mention Arrow-Hurwicz method and Uzawa method [2], whose differential form based on the variational formulation of the original problem and dual theory [3] allows one to separate the process of finding unknowns and to solve in this way the issue on the absence of the equation for the pressure. The approach we consider below is close in spirit to the methods in [4], [5].

I.I. Golichev, T.R. Sharipov, N.I. Luchnikova, Gradient methods for SOlving Stokes Problem.

(c) Golichev I.I., Sharipov T.R., Luchnikova N.I. 2016.

Submitted December 9, 2015. 
Together with the problem on minimization of function $J(\mathbf{u})$ in space $\boldsymbol{L}_{2}(\Omega)$, we shall also consider problems on minimization of $J(\nabla u)$ in spaces $H^{1}(\Omega)$ and $L_{2}(\Omega)$, in which pressure $p$ serves as control $u$. Since the gradients of the functionals in these cases have different forms, it is convenient to denote them accordingly to the considered problems:

Problem I. Find the minimum of the functional $J_{0}(\mathbf{u})=\|\operatorname{div} \mathbf{v}(\mathbf{u})\|_{L_{2}(\Omega)}^{2} / 2$ on the set $U_{0}=G(\Omega)$, where $\mathbf{v}(\mathbf{u})$ is the solution to problem (4).

Problem II. Find the minimum of the functional $J_{1}(u)=\|\operatorname{div} \mathbf{v}(u)\|_{L_{2}(\Omega)}^{2} / 2$ on the set $U_{1}=\left\{u \in H^{1}(\Omega):(u, 1)_{L_{2}(\Omega)}=0\right\}$, where $\mathbf{v}(u)$ is the solution to the problem

$$
-\nu \Delta \mathbf{v}(u)=\mathbf{f}-\nabla u,\left.\mathbf{v}(u)\right|_{S}=0 .
$$

Problem III. Find the minimum of the functional $J_{2}(u)=\|\operatorname{div} \mathbf{v}(u)\|_{L_{2}(\Omega)}^{2} / 2$ on the set $U_{2}=\left\{u \in L_{2}(\Omega):(u, 1)_{L_{2}(\Omega)}=0\right\}$, where $\mathbf{v}(u)$ is the generalized solution to problem (5).

We shall seek the solutions to Problems I-III be the gradient projection method [6], [7]:

$$
u_{k+1}=P_{U_{l}}\left(u_{k}-\alpha_{k} J_{l}^{\prime}\left(u_{k}\right)\right), l=0,1,2,
$$

where $P_{U_{l}}$ is the operator of projection on set $U_{l}$, and $J_{l}^{\prime}\left(u_{k}\right)$ is the gradient of the functional $J_{l}\left(u_{k}\right)$ at point $u_{k}^{1}$.

In what follows we shall show that

$$
\begin{aligned}
& J_{0}^{\prime}(\mathbf{u})=\mathbf{w}(\mathbf{u}), \\
& J_{1}^{\prime}(u)=\rho(\mathbf{w}(u)), \\
& J_{2}^{\prime}(u)=-\operatorname{div} \mathbf{w}(u),
\end{aligned}
$$

where $\mathbf{w}(\cdot)$ is the dual state of system (4) introduced for all Problems as the solution to the problem

$$
-\nu \Delta \mathbf{w}(\cdot)=\nabla \operatorname{div} \mathbf{v}(\cdot),\left.\mathbf{w}(\cdot)\right|_{S}=0
$$

while $\rho(\mathbf{w})$ is determined by the orthogonal expansion of the vector $\mathbf{w}(u)=\nabla \rho(\mathbf{w})+\vec{\varphi}$ into the gradient and solenoidal components.

Let us find projection operators for all considered Problems and let us show that Problems I and II are equivalent.

We note that in [4] an analogue of Problem II for the generalized Stokes problem was considered, while for constructing an iteration process, the general theory of self-adjoint operator equations was employed. We use a test calculation from this work to verify our calculations by formula (6). The method of complete and incomplete separation of boundary conditions [5], where the theory of boundary Poincaré-Steklov operators is employed, leads one to differential iteration processes close by structure to those in the present work.

Hereafter by $\stackrel{\circ}{\mathbf{v}}(\mathbf{u})$ we denote the solution to problem (4) as $\mathbf{f}=0$, while by $\stackrel{\circ}{\mathbf{v}}(u)$ we denote the solution to problem (5) as $\mathbf{f}=0$.

\section{DifFERENTIABILITY OF FUnCTIONAL $J_{0}(\mathbf{u})$}

We denote by $L$ the operator $L(\mathbf{z})=-\nu \Delta \mathbf{z}$ on the set $\boldsymbol{H}_{0}^{2}(\Omega)=\left\{\mathbf{z} \in \boldsymbol{H}^{2}(\Omega):\left.\mathbf{z}\right|_{S}=0\right\}$. We note that the range of operator $L$ coincides with space $\boldsymbol{L}_{2}(\Omega)$. Indeed, let $\mathbf{f}$ be an arbitrary element in $\boldsymbol{L}_{2}(\Omega)$; then in accordance with [8, Ch. II, Sect. 7, Thm. 7.1] the problem

$$
L(\mathbf{z})=\mathbf{f}
$$

has the unique solution in $\boldsymbol{H}_{0}^{2}(\Omega)$.

Employing the second energy inequality [8, Ch. II, Sect. 6, Formula (6.29) $]^{2}$

$$
\|\mathbf{z}\|_{H^{2}(\Omega)} \leqslant c_{1}\|\Delta \mathbf{z}\|_{L_{2}(\Omega)}
$$

\footnotetext{
${ }^{1}$ For the sake of the generalized notation, we do not use bold fond for vector $u$ in Problem I.

${ }^{2}$ If domain $\Omega$ is convex, then constant $c_{1}$ is equal to one.
} 
In view of the obvious for problem (11) identity $\|\Delta \mathbf{z}\|_{L_{2}(\Omega)}=\nu^{-1}\|\mathbf{f}\|_{L_{2}(\Omega)}$, we obtain the estimate

$$
\|\mathbf{z}\|_{H^{2}(\Omega)} \leqslant c_{1} \nu^{-1}\|\mathbf{f}\|_{L_{2}(\Omega)},
$$

which will be employed in what follows.

Let $\mathbf{u}$ and $\mathbf{h}$ be arbitrary elements in $U_{0}$. Taking into consideration the definition of operator $L$, it is easy to see that $\mathbf{v}(\mathbf{u}+\mathbf{h})-\mathbf{v}(\mathbf{u})=\stackrel{\circ}{\mathbf{v}}(\mathbf{h})$, where $\stackrel{\circ}{\mathbf{v}}(\mathbf{h})=L^{-1}(-\mathbf{h})$. Hence,

$$
\begin{aligned}
J_{0}(\mathbf{u}+\mathbf{h})-J_{0}(\mathbf{u}) & =\frac{1}{2}\|\operatorname{div} \mathbf{v}(\mathbf{u}+\mathbf{h})\|_{L_{2}(\Omega)}^{2}-\frac{1}{2}\|\operatorname{div} \mathbf{v}(\mathbf{u})\|_{L_{2}(\Omega)}^{2} \\
& =(\operatorname{div} \mathbf{v}(\mathbf{u}), \operatorname{div} \stackrel{\circ}{\mathbf{v}}(\mathbf{h}))_{L_{2}(\Omega)}+\frac{1}{2}\|\operatorname{div} \stackrel{\circ}{\mathbf{v}}(\mathbf{h})\|_{L_{2}(\Omega)}^{2} .
\end{aligned}
$$

We transform the first term in the right hand of the last identity by integrating by parts and employing the condition $\left.\stackrel{\mathbf{v}}{(\mathbf{h})}\right|_{S}=0$ and the self-adjointness of operator $L$ :

$$
(\operatorname{div} \mathbf{v}(\mathbf{u}), \operatorname{div} \stackrel{\circ}{\mathbf{v}}(\mathbf{h}))_{L_{2}(\Omega)}=-\left(\nabla \operatorname{div} \mathbf{v}(\mathbf{u}), L^{-1}(-\mathbf{h})\right)_{\boldsymbol{L}_{2}(\Omega)}=\left(L^{-1} \nabla \operatorname{div} \mathbf{v}(\mathbf{u}), \mathbf{h}\right)_{\boldsymbol{L}_{2}(\Omega)} .
$$

Thus, in view of the notation $\mathbf{w}(\mathbf{u})=L^{-1} \nabla \operatorname{div} \mathbf{v}(\mathbf{u})$, identity (14) becomes

$$
J_{0}(\mathbf{u}+\mathbf{h})-J_{0}(\mathbf{u})=(\mathbf{w}(\mathbf{u}), \mathbf{h})_{L_{2}(\Omega)}+\frac{1}{2}\|\operatorname{div} \stackrel{\circ}{\mathbf{v}}(\mathbf{h})\|_{L_{2}(\Omega)}^{2} .
$$

Multiplying both sides of (11) by $\mathbf{z}$ and integrating by parts, we obtain the relation

$$
\nu\|\nabla \mathbf{z}\|_{\boldsymbol{L}_{2}(\Omega)}^{2} \leqslant\|\mathbf{f}\|_{\boldsymbol{L}_{2}(\Omega)}\|\mathbf{z}\|_{\boldsymbol{L}_{2}(\Omega)},
$$

which together with Fridrichs inequality

$$
\|\mathbf{z}\|_{L_{2}(\Omega)} \leqslant c_{0}\|\nabla \mathbf{z}\|_{L_{2}(\Omega)}
$$

yield:

$$
\|\nabla \mathbf{z}\|_{L_{2}(\Omega)} \leqslant c_{0} \nu^{-1}\|\mathbf{f}\|_{L_{2}(\Omega)}
$$

By (17) and the easily checked inequality

$$
\|\operatorname{div} \mathbf{z}\|_{L_{2}(\Omega)} \leqslant \sqrt{n}\|\nabla \mathbf{z}\|_{L_{2}(\Omega)}
$$

we get that

$$
\|\operatorname{div} \stackrel{\circ}{\mathbf{v}}(\mathbf{h})\|_{L_{2}(\Omega)} \leqslant \sqrt{n} c_{0} \nu^{-1}\|\mathbf{h}\|_{L_{2}(\Omega)}
$$

Hence, the principal linear part of the increment of functional $J_{0}(\mathbf{u})$ is determined by the expression $(\mathbf{w}(\mathbf{u}), \mathbf{h})_{L_{2}(\Omega)}$.

Let us show that the gradient $J_{0}^{\prime}(\mathbf{u})$ satisfies Lipschitz condition. Let $\mathbf{u}^{(1)}, \mathbf{u}^{(2)} \in U_{0}$, and $\mathbf{w}^{(1)}, \mathbf{w}^{(2)}$ are the corresponding solutions to problem 10$)$. We observe that

$$
L\left(\mathbf{w}^{(1)}-\mathbf{w}^{(2)}\right)=\nabla \operatorname{div} \mathbf{v}\left(\mathbf{u}^{(1)}\right)-\nabla \operatorname{div} \mathbf{v}\left(\mathbf{u}^{(2)}\right)=\nabla \operatorname{div} \stackrel{\circ}{\mathbf{v}}\left(\mathbf{u}^{(1)}-\mathbf{u}^{(2)}\right),
$$

that by inequalities (16), 17) imply

$$
\left\|\mathbf{w}^{(1)}-\mathbf{w}^{(2)}\right\|_{\boldsymbol{L}_{2}(\Omega)} \leqslant c_{0}^{2} \nu^{-1}\left\|\nabla \operatorname{div} \stackrel{\circ}{\mathbf{v}}\left(\mathbf{u}^{(1)}-\mathbf{u}^{(2)}\right)\right\|_{\boldsymbol{L}_{2}(\Omega)} .
$$

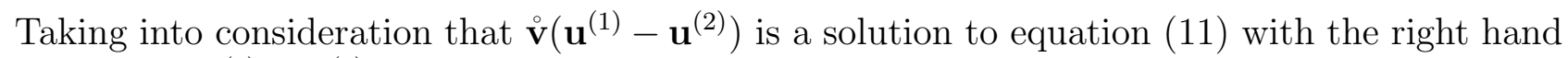
side $\mathbf{f}=-\left(\mathbf{u}^{(1)}-\mathbf{u}^{(2)}\right)$ and this solution satisfies estimate $(13)$, by 20 we obtain:

$$
\begin{aligned}
\left\|J_{0}^{\prime}\left(\mathbf{u}^{(1)}\right)-J_{0}^{\prime}\left(\mathbf{u}^{(2)}\right)\right\|_{\boldsymbol{L}_{2}(\Omega)} & =\left\|\mathbf{w}^{(1)}-\mathbf{w}^{(2)}\right\|_{\boldsymbol{L}_{2}(\Omega)} \leqslant \sqrt{n} c_{0}^{2} \nu^{-1}\left\|\stackrel{\circ}{\mathbf{v}}\left(\mathbf{u}^{(1)}-\mathbf{u}^{(2)}\right)\right\|_{\boldsymbol{H}^{2}(\Omega)} \\
& \leqslant \sqrt{n} c_{0}^{2} c_{1} \nu^{-2}\left\|\mathbf{u}^{(1)}-\mathbf{u}^{(2)}\right\|_{\boldsymbol{L}_{2}(\Omega)} .
\end{aligned}
$$

Thus, we have proved the following theorem.

Theorem 1. Let $\boldsymbol{f} \in \boldsymbol{L}_{2}(\Omega)$, where $\Omega$ is a bounded domain with a boundary $S \in C^{2}$. Then functional $J_{0}(\boldsymbol{u})$ is Fréchet differentiable on $U_{0}$, and its gradient is determined by formula (7) and satisfies Lipschitz condition with constant $L_{0}=\sqrt{n} c_{0}^{2} c_{1} \nu^{-2}$, where $c_{0}$ and $c_{1}$ are constants in inequalities (16), 12. 


\section{Differentiability of FUnCtional $J_{1}(u)$}

On set $U_{1}$ we introduce a metric equivalent to the metric in space $H^{1}(\Omega)$ via the scalar product $(a, b)_{H_{0}^{1}(\Omega)}=(\nabla a, \nabla b)_{L_{2}(\Omega)}$.

Let $u$ and $h$ be arbitrary elements in $U_{1}$. Similar to $(15)$ it is easy to see that

$$
J_{1}(u+h)-J_{1}(u)=(\mathbf{w}(u), \nabla h)_{L_{2}(\Omega)}+\frac{1}{2}\|\operatorname{div} \stackrel{\circ}{\mathbf{v}}(h)\|_{L_{2}(\Omega)}^{2}, \stackrel{\circ}{\mathbf{v}}(h)=L^{-1}(-\nabla h),
$$

and in view of the expansion of the vector $\mathbf{w}(u)=P_{G(\Omega)} \mathbf{w}(u)+\vec{\varphi}$ into the gradient and solenoidal components $(\operatorname{div} \vec{\varphi}=0)$,

$$
(\mathbf{w}(u), \nabla h)_{\boldsymbol{L}_{2}(\Omega)}=\left(P_{G(\Omega)} \mathbf{w}(u), \nabla h\right)_{\boldsymbol{L}_{2}(\Omega)} .
$$

It is known [9] that for all $\mathbf{d} \in \boldsymbol{L}_{2}(\Omega)$ we have $P_{G(\Omega)} \mathbf{d}=\nabla \rho$, where $\rho$ is the solution to the Neumann problem

$$
\Delta \rho=\operatorname{div} \mathbf{d},\left.\frac{\partial \rho}{\partial \vec{n}}\right|_{S}=\left.(\mathbf{d} \cdot \vec{n})\right|_{S}
$$

It follows from (22), (23), (24):

$$
J_{1}(u+h)-J_{1}(u)=(\rho(\mathbf{w}), h)_{H_{0}^{1}(\Omega)}+\frac{1}{2}\|\operatorname{div} \stackrel{\circ}{\mathbf{v}}(h)\|_{L_{2}(\Omega)}^{2},
$$

where $\rho(\mathbf{w})$ is the solution to the problem

$$
\Delta \rho(\mathbf{w})=\operatorname{div} \mathbf{w}(u),\left.\frac{\partial \rho(\mathbf{w})}{\partial \vec{n}}\right|_{S}=\left.(\mathbf{w}(u) \cdot \vec{n})\right|_{S} .
$$

By analogy with 19 we have the estimate

$$
\|\operatorname{div} \stackrel{\circ}{\mathbf{v}}(h)\|_{L_{2}(\Omega)} \leqslant \sqrt{n} c_{0} \nu^{-1}\|\nabla h\|_{L_{2}(\Omega)}=\sqrt{n} c_{0} \nu^{-1}\|h\|_{H_{0}^{1}(\Omega)} .
$$

This implies that the principal linear part of the functional $J_{1}(u)$ is determined by the expression $(\rho(\mathbf{w}), h)_{H_{0}^{1}(\Omega)}$.

Let us show that the gradient $J_{1}^{\prime}(u)$ satisfies the Lipschitz condition. Let $u^{(1)}$ and $u^{(2)}$ belong to $U_{1}$, and $\mathbf{w}^{(1)}$ and $\mathbf{w}^{(2)}$ are the associated solutions to problem (10). Then

$$
\begin{aligned}
\left\|J_{1}^{\prime}\left(u^{(1)}\right)-J_{1}^{\prime}\left(u^{(2)}\right)\right\|_{H_{0}^{1}(\Omega)} & =\left\|\rho\left(u^{(1)}\right)-\rho\left(u^{(2)}\right)\right\|_{H_{0}^{1}(\Omega)}=\left\|\nabla \rho\left(u^{(1)}\right)-\nabla \rho\left(u^{(2)}\right)\right\|_{L_{2}(\Omega)} \\
& =\left\|P_{G(\Omega)} \mathbf{w}^{(1)}-P_{G(\Omega)} \mathbf{w}^{(1)}\right\|_{L_{2}(\Omega)} \leqslant\left\|\mathbf{w}^{(1)}-\mathbf{w}^{(2)}\right\|_{L_{2}(\Omega)} .
\end{aligned}
$$

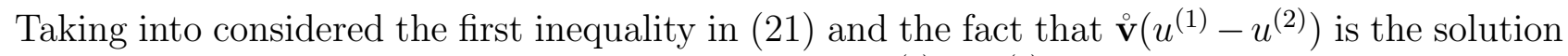
to equation (11) with the right hand side $\mathbf{f}=-\nabla\left(u^{(1)}-u^{(2)}\right)$ and taking into consideration estimate (13), we obtain

$$
\begin{aligned}
\left\|J_{1}^{\prime}\left(u^{(1)}\right)-J_{1}^{\prime}\left(u^{(2)}\right)\right\|_{H_{0}^{1}(\Omega)} & \leqslant\left\|\mathbf{w}^{(1)}-\mathbf{w}^{(2)}\right\|_{L_{2}(\Omega)} \leqslant \sqrt{n} c_{0}^{2} c_{1} \nu^{-2}\left\|\nabla u^{(1)}-\nabla u^{(2)}\right\|_{L_{2}(\Omega)} \\
& =\sqrt{n} c_{0}^{2} c_{1} \nu^{-2}\left\|u^{(1)}-u^{(2)}\right\|_{H_{0}^{1}(\Omega)} .
\end{aligned}
$$

Theorem 2. Suppose that the assumptions of Theorem 1 hold true. Then functional $J_{1}(u)$ is Fréchet differentiable on $U_{1}$, its gradient is determined by the formula (8) and it satisfies the Lipschitz condition with constant $L_{0}$.

If in Problems I and II the initial approximations in iteration processes (6) as $l=0$ and $l=1$ are related by the identity $\mathbf{u}_{0}=\nabla u_{0}$, then $\mathbf{u}_{k}=\nabla u_{k}$ for all $k=1,2, \ldots$ Indeed, if $\mathbf{u}_{k}=\nabla u_{k}$, then it is obvious that $\mathbf{v}\left(\mathbf{u}_{k}\right)=\mathbf{v}\left(u_{k}\right)$ and $\mathbf{w}\left(\mathbf{u}_{k}\right)=\mathbf{w}\left(u_{k}\right)$, and hence

$$
\begin{aligned}
\mathbf{u}_{k+1} & =P_{G(\Omega)}\left(\mathbf{u}_{k}-\alpha_{k} J_{0}^{\prime}\left(\mathbf{u}_{k}\right)\right)=\mathbf{u}_{k}-\alpha_{k} P_{G(\Omega)} \mathbf{w}\left(\mathbf{u}_{k}\right) \\
& =\mathbf{u}_{k}-\alpha_{k} \nabla \rho_{k}\left(u_{k}\right)=\nabla\left(u_{k}-\alpha_{k} \rho_{k}\left(u_{k}\right)\right)=\nabla u_{k+1} .
\end{aligned}
$$

Hence, Problem I and II can be regarded as equivalent. 


\section{Differentiability of FUnCtional $J_{2}(u)$}

Let $u$ and $h$ be arbitrary elements in $U_{2}$. In proving formula (9), we can not use directly integration by parts as in the previous sections since here we do not guarantee the belonging of functions $\mathbf{v}(u)$ and $\mathbf{w}(u)$ to space $\boldsymbol{H}^{2}(\Omega)$. We make use the passage to the limit. We choose sequences $\left\{u_{n}\right\},\left\{h_{n}\right\}$ in $U_{1}$ so that $u_{n} \rightarrow u, h_{n} \rightarrow h$ in $L_{2}(\Omega)$ as $n \rightarrow \infty$.

Identity 22 holds true on sequences $\left\{u_{n}\right\},\left\{h_{n}\right\}$ :

$$
J_{2}\left(u_{n}+h_{n}\right)-J_{2}\left(u_{n}\right)=\left(\mathbf{w}\left(u_{n}\right), \nabla h_{n}\right)_{L_{2}(\Omega)}+\frac{1}{2}\left\|\operatorname{div} \stackrel{\circ}{\mathbf{v}}\left(h_{n}\right)\right\|_{L_{2}(\Omega)}^{2} .
$$

Let us show that $\operatorname{div} \mathbf{w}\left(u_{n}\right) \rightarrow \operatorname{div} \mathbf{w}(u), \operatorname{div} \stackrel{\circ}{\mathbf{v}}\left(h_{n}\right) \rightarrow \operatorname{div} \stackrel{\circ}{\mathbf{v}}(h)$ as $n \rightarrow \infty$ in $L_{2}(\Omega)$.

Multiplying equation (5) by $\mathbf{v}$ and integrating by parts, we obtain the identity

$$
\nu\|\nabla \mathbf{v}\|_{\boldsymbol{L}_{2}(\Omega)}^{2}=(\mathbf{f}, \mathbf{v})_{\boldsymbol{L}_{2}(\Omega)}+(u, \operatorname{div} \mathbf{v})_{L_{2}(\Omega)} .
$$

Applying then inequalities (18) and (16), we arrive at the inequality

$$
\|\nabla \mathbf{v}\|_{L_{2}(\Omega)} \leqslant \nu^{-1}\left(c_{0}\|\mathbf{f}\|_{L_{2}(\Omega)}+\sqrt{n}\|u\|_{L_{2}(\Omega)}\right) .
$$

The difference $\mathbf{v}\left(u^{(1)}\right)-\mathbf{v}\left(u^{(2)}\right)=\stackrel{\circ}{\mathbf{v}}\left(u^{(1)}-u^{(2)}\right)$ solves problem (5) as $\mathbf{f}=0$ and $u=u^{(1)}-u^{(2)}$. Then by inequality 27

$$
\left\|\nabla \stackrel{\circ}{\mathbf{v}}\left(u^{(1)}-u^{(2)}\right)\right\|_{\boldsymbol{L}_{2}(\Omega)} \leqslant \nu^{-1} \sqrt{n}\left\|u^{(1)}-u^{(2)}\right\|_{L_{2}(\Omega)} .
$$

In the same way, using estimates (18), (28), we obtain the inequality

$$
\begin{aligned}
\left\|\nabla \mathbf{w}\left(u^{(1)}\right)-\nabla \mathbf{w}\left(u^{(2)}\right)\right\|_{L_{2}(\Omega)} & \leqslant \nu^{-1} \sqrt{n}\left\|\operatorname{div} \mathbf{v}\left(u^{(1)}\right)-\operatorname{div} \mathbf{v}\left(u^{(2)}\right)\right\|_{L_{2}(\Omega)} \\
& \leqslant \nu^{-1} n\left\|\nabla \dot{\mathbf{v}}\left(u^{(1)}-u^{(2)}\right)\right\|_{L_{2}(\Omega)} \leqslant \nu^{-2} n^{3 / 2}\left\|u^{(1)}-u^{(2)}\right\|_{L_{2}(\Omega)} .
\end{aligned}
$$

It follows from two last estimates and inequality (17) that we can pass to the limit as $n \rightarrow \infty$ in relations (26). As the result, we obtain

$$
\begin{aligned}
J_{2}(u+h)-J_{2}(u) & =(\mathbf{w}(u), \nabla h)_{L_{2}(\Omega)}+\frac{1}{2}\|\operatorname{div} \stackrel{\circ}{\mathbf{v}}(h)\|_{L_{2}(\Omega)}^{2} \\
& =(-\operatorname{div} \mathbf{w}(u), h)_{L_{2}(\Omega)}+\frac{1}{2}\|\operatorname{div} \stackrel{\circ}{\mathbf{v}}(h)\|_{L_{2}(\Omega)}^{2},
\end{aligned}
$$

and in view of estimates 18$),(28)$

$$
\|\operatorname{div} \stackrel{\circ}{\mathbf{v}}(h)\|_{L_{2}(\Omega)} \leqslant \nu^{-1} n\|h\|_{L_{2}(\Omega)} .
$$

The Lipschitz condition for $J_{2}^{\prime}(u)$ can be established by applying inequality $(29)$ :

$$
\begin{aligned}
\left\|J_{2}^{\prime}\left(u^{(1)}\right)-J_{2}^{\prime}\left(u^{(1)}\right)\right\|_{L_{2}(\Omega)} & =\left\|\operatorname{div} \mathbf{w}\left(u^{(1)}-u^{(2)}\right)\right\|_{L_{2}(\Omega)} \\
& \leqslant \sqrt{n}\left\|\nabla \mathbf{w}\left(u^{(1)}-u^{(2)}\right)\right\|_{L_{2}(\Omega)} \leqslant \nu^{-2} n^{2}\left\|u^{(1)}-u^{(2)}\right\|_{L_{2}(\Omega)} .
\end{aligned}
$$

Thus, we arrive at the following theorem

Theorem 3. Suppose that the assumptions of Theorem 2 hold true. Then functional $J_{2}(u)$ is Fréchet differentiable on $U_{2}$, its gradient is determined by formula (9) and satisfies the Lipschitz condition with constant $L_{2}=\nu^{-2} n^{2}$.

\section{Modified Steepest Descent GRAdient Method}

There exist various ways of choosing $\alpha_{k}$ (see [6], [7]). The fastest convergence is given by the steepest descent method, in which $\alpha_{k}$ is determined by the condition

$$
f_{k}\left(\alpha_{k}\right)=\min _{\alpha>0} f_{k}(\alpha), f_{k}(\alpha)=J_{l}\left(P_{U_{l}}\left(u_{k}-\alpha J_{l}^{\prime}\left(u_{k}\right)\right)\right), l=0,1,2 .
$$

In the general case, at each step of descent we need to solve one-parametrical optimization problem (30). However, in the considered problem we can find explicit formulae for parameters 
$\alpha_{k}$ employing that sets $U_{l}$ are subspaces of the corresponding Hilbert spaces and therefore, projection operators $P_{l}$ are linear.

If $u_{k} \in U_{l}$, then $P_{U_{l}}\left(u_{k}\right)=u_{k}$ and hence, in view of the notation $d_{k}=P_{U_{l}}\left(J_{l}^{\prime}\left(u_{k}\right)\right)$, we have

$$
\begin{aligned}
f_{k}(\alpha) & =J_{l}\left(u_{k}-\alpha d_{k}\right)=\left\|\operatorname{div} \mathbf{v}\left(u_{k}\right)-\alpha \operatorname{div} \stackrel{\circ}{\mathbf{v}}\left(d_{k}\right)\right\|_{L_{2}(\Omega)}^{2} / 2 \\
& =\left\|\operatorname{div} \mathbf{v}\left(u_{k}\right)\right\|_{L_{2}(\Omega)}^{2} / 2-\alpha\left(\operatorname{div} \mathbf{v}\left(u_{k}\right), \operatorname{div} \stackrel{\circ}{\mathbf{v}}\left(d_{k}\right)\right)_{L_{2}(\Omega)}+\alpha^{2}\left\|\operatorname{div} \mathbf{v}\left(d_{k}\right)\right\|_{L_{2}(\Omega)}^{2} / 2 .
\end{aligned}
$$

Hence, the point of the minimum is

$$
\alpha_{k}^{\prime}=\frac{\left(\operatorname{div} \mathbf{v}\left(u_{k}\right), \operatorname{div} \stackrel{\circ}{\mathbf{v}}\left(d_{k}\right)\right)_{L_{2}(\Omega)}}{\left\|\operatorname{div} \stackrel{\circ}{\mathbf{v}}\left(d_{k}\right)\right\|_{L_{2}(\Omega)}^{2}},
$$

and (6) becomes

$$
u_{k+1}=u_{k}-\alpha_{k}^{\prime} d_{k}
$$

Application of the steepest descent method for the considered problems faces the trouble related to the fact that functionals $J_{l}(u)$ do not satisfy the boundedness condition for Lebesgue set $M_{l, c}=\left\{u \in U_{l}: J(u) \leqslant c\right\}$, which used in the proof of the convergence of the steepest descent method. This problem turns out to be possible to be overcomed if we choose $\alpha_{k}$ by the formula

$$
\alpha_{k}=\min \left[\alpha_{k}^{\prime}, \gamma\right]
$$

where $\gamma$ is the parameter of the method, and $\alpha_{k}^{\prime}$ is determined by formula (31) as in the steepest descent method.

Since the proposed method can be used in other optimization problems, we formulate the statement as a theorem in an abstract Hilbert space $H$. We introduce the notations: $J_{*}=$ $\inf _{U} J(u), U \subset H, U_{*}=\left\{u \in U: J(u)=J_{*}\right\}, C^{1,1}(U)$ is the set of differentiable functionals, whose gradients satisfy the Lipschitz condition.

Theorem 4. 1 Let $U$ be a convex closed set in a Hilbert space $H$ with norm $\|\cdot\|, J(u) \in$ $C^{1,1}(u)$ be a convex functional $\left(J_{*}>-\infty\right)$, set $U_{*}$ be non-empty and bounded, sequence $\left\{u_{k}\right\}_{k=0}^{\infty}$ be introduced by formulae (33), (6) $)^{2}$ and the condition

$$
\begin{aligned}
& \sum_{k=0}^{\infty}\left\|J^{\prime}\left(u_{k}\right)\right\|^{2} \leqslant b_{1}, \\
& 0<\alpha_{k}<b_{2} .
\end{aligned}
$$

hold true. Then sequence $\left\{u_{k}\right\}_{k=0}^{\infty}$ minimizes function $J(u)$ on $U$ and converges weakly to set $U_{*}$ in $H$.

Proof. We denote $\rho\left(u, U_{*}\right)=\min _{v \in U_{*}}\|u-v\|$. Then by the definition of the projection operator

$$
\begin{aligned}
\rho^{2}\left(u_{k+1}, U_{*}\right) & =\left\|u_{k+1}-P_{U_{*}}\left(u_{k+1}\right)\right\|^{2} \leqslant\left\|u_{k+1}-P_{U_{*}}\left(u_{k}\right)\right\|^{2} \\
& =\left\|P_{U}\left(u_{k}-\alpha_{k} J^{\prime}\left(u_{k}\right)\right)-P_{U}\left(P_{U_{*}}\left(u_{k}\right)\right)\right\|^{2} \leqslant\left\|u_{k}-\alpha_{k} J^{\prime}\left(u_{k}\right)-P_{U_{*}}\left(u_{k}\right)\right\|^{2} \\
& =\rho^{2}\left(u_{k}, U_{*}\right)+\alpha_{k}^{2}\left\|J^{\prime}\left(u_{k}\right)\right\|^{2}-2 \alpha_{k}\left(J^{\prime}\left(u_{k}\right), u_{k}-P_{U_{*}}\left(u_{k}\right)\right) .
\end{aligned}
$$

Employing the convexity criteria of a differentiable functional on convex set $U[6]: J(u)-J(v) \geqslant$ $\left(J^{\prime}(v), u-v\right), \forall u, v \in U$, and letting $v=u_{k}, u=P_{U_{*}}\left(u_{k}\right)$, we obtain

$$
\left(J^{\prime}\left(u_{k}\right), u_{k}-P_{U_{*}}\left(u_{k}\right)\right) \geqslant J\left(u_{k}\right)-J\left(P_{U_{*}}\left(u_{k}\right)\right)=J\left(u_{k}\right)-J_{*} \geqslant 0 .
$$

Hence, inequality (35) becomes

$$
\rho^{2}\left(u_{k+1}, U_{*}\right)-\rho^{2}\left(u_{k}, U_{*}\right) \leqslant \alpha_{k}^{2}\left\|J^{\prime}\left(u_{k}\right)\right\|^{2} .
$$

\footnotetext{
${ }^{1}$ This theorem and its prooof is provided in work [10]. However, the statement that if domain $U$ is a subspace or a space then condition (34) can be omitted, was proven for a particular functional. In the present work this is proved for each convex functional $J(u) \in C^{1,1}(U)$.

${ }^{2}$ If functional $J(u)$ has no subscript, the same is assumed in formula (6).
} 
Summing up the last inequality from 0 to $m-1,(m>0)$, and taking into consideration condition (34), we obtain

$$
\rho^{2}\left(u_{m}, U_{*}\right) \leqslant \sum_{k=0}^{m-1} \alpha_{k}^{2}\left\|J^{\prime}\left(u_{k}\right)\right\|^{2}+\rho^{2}\left(u_{0}, U_{*}\right) \leqslant b_{2}^{2} b_{1}+\rho^{2}\left(u_{0}, U_{*}\right) .
$$

Hence, sequence $\left\{u_{k}\right\}_{k=0}^{\infty}$ is bounded in $H$, while it follows from condition (34) that $\lim _{k \rightarrow \infty}\left\|J^{\prime}\left(u_{k}\right)\right\|=0$. Then inequality (36) implies that sequence $\left\{u_{k}\right\}_{k=0}^{\infty}$ minimize functional $J(u)$. Thus, sequence $\left\{u_{k}\right\}_{k=0}^{\infty}$ is bounded and minimizes $J(u)$ on $U$.

We denote by $W$ the set of convex combinations of sequence $\left\{u_{k}\right\}_{k=0}^{\infty}$, that is, the set of points $u$ represented as:

$$
u=\sum_{k=0}^{\infty} \alpha_{k} u_{k}, \alpha_{k} \geqslant 0, \sum_{k=0}^{\infty} \alpha_{k}=1 .
$$

Employing [7, Ch. 4, Sect. 1, Thm. 5], it is easy to show that $W \subset U$ and since $U$ is a closed set, closure $\bar{W}$ of set $W$ also belongs to $U$.

Sequence $\left\{u_{k}\right\}_{k=0}^{\infty}$ minimizes function $J(u)$ on $U$ and therefore, it minimizes $J(u)$ on $\bar{W}$. This implies that $J_{*}(\bar{W})=\inf _{u \in \bar{W}} J(u)=J_{*}=\inf _{u \in U} J(u), \bar{W}_{*}=\left\{u \in \bar{W}: J(u)=J_{*}\right\} \in U_{*}$. The boundedness of sequence $\left\{u_{k}\right\}_{k=0}^{\infty}$ implies the boundedness of set $\bar{W}$. In accordance with [․ 1, Sect. 3, Thm. 6], a convex lower semibounded functional $J(u)$ on a bounded convex closed set $U$ in a reflexive Banach space has a non-empty set of minimums $U_{*}$ and each minimizing sequence $\left\{u_{k}\right\}_{k=0}^{\infty}$ converges weakly to $U_{*}$. The weak convergence of sequence $\left\{u_{k}\right\}_{k=0}^{\infty}$ to $\bar{W}_{*}$ implies its weak convergence to $U_{*}$. The proof is complete.

Remark 1. If set $\bar{W}$ is compact, then a strong convergence hold true. Here we can employ [6, Ch. 1, Sect. 3, Thm. 1].

Remark 2. If $U$ is a subspace of Hilbert space $H, P_{U}$ is an operator orthogonal projection on this subspace, then $u_{k+1}=u_{k}-P_{U} J^{\prime}\left(u_{k}\right)$. In this case relation (35) can be written as

$$
\rho^{2}\left(u_{k+1}, U_{*}\right)=\rho^{2}\left(u_{k}, U_{*}\right)+\alpha_{k}^{2}\left\|P_{U} J^{\prime}\left(u_{k}\right)\right\|^{2}-2 \alpha_{k}\left(P_{U} J^{\prime}\left(u_{k}\right), u_{k}-P_{U_{*}}\left(u_{k}\right)\right) .
$$

Taking into consideration that $\left(P_{U} J^{\prime}\left(u_{k}\right), u_{k}-P_{U_{*}}\left(u_{k}\right)\right)=\left(J^{\prime}\left(u_{k}\right), u_{k}-P_{U_{*}}\left(u_{k}\right)\right)$, it is easy to see that the statements of the theorem holds true if instead of condition (34), the condition

$$
\sum_{k=0}^{\infty}\left\|P_{U} J^{\prime}\left(u_{k}\right)\right\|^{2}<b_{1}
$$

holds true.

Theorem 5. Let $U$ be a subspace or a Hilbert space $H, J(u) \in C^{1,1}(U)$ be a convex functional, set $U_{*}$ is non-empty and bounded, sequence $\left\{u_{k}\right\}_{k=0}^{\infty}$ is defined by formulae (33), (6). Then sequence $\left\{u_{k}\right\}$ minimizes functional $J(u)$ on $U$ and converges to set $U_{*}$ weakly in $H$.

Proof. Let us show that if $J(u) \in C^{1,1}(U)$, where $U$ is either a subspace or space $H$ and parameter $\alpha_{k}$ is determined by formulae (33), (30), then condition (39) holds true and therefore, the statements of the theorem hold without condition (34). In order to do it, we make use of a known inequality valid for functions $C^{1,1}(u)$ (see [7, Ch. 2, Sect. 3, Lm. 1]):

$$
\left|J(u)-J(v)-\left(J^{\prime}(v), u-v\right)\right| \leqslant L\|u-v\|^{2} / 2 \forall u, v \in U,
$$

where $L$ is the Lipschitz constant for the gradient $J^{\prime}(u)$ of functional $J(u)$.

Letting $v=u_{k}, u=u_{k+1}^{\alpha}=u_{k}-\alpha P_{U} J^{\prime}\left(u_{k}\right)$, we obtain

$$
\begin{gathered}
J\left(u_{k}\right)-J\left(u_{k+1}^{\alpha}\right)=J\left(u_{k}\right)-J\left(u_{k}-\alpha P_{U} J^{\prime}\left(u_{k}\right)\right) \\
\geqslant \alpha\left(J^{\prime}\left(u_{k}\right), P_{U} J^{\prime}\left(u_{k}\right)\right)-\alpha^{2} L\left\|P_{U} J^{\prime}\left(u_{k}\right)\right\|^{2} / 2 .
\end{gathered}
$$


Taking into consideration that $P_{U}$ is the operator of orthogonal projection on the subspace, we obtain that $\left(J^{\prime}\left(u_{k}\right), P_{U} J^{\prime}\left(u_{k}\right)\right)=\left\|P_{U} J^{\prime}\left(u_{k}\right)\right\|$, while inequality (41) implies

$$
J\left(u_{k}\right)-J\left(u_{k+1}^{\alpha}\right) \geqslant \alpha(1-\alpha L / 2)\left\|P_{U} J^{\prime}\left(u_{k}\right)\right\|^{2} .
$$

Letting $\alpha=1 / L$, we obtain

$$
J\left(u_{k}\right)-J\left(u_{k+1}^{\alpha}\right) \geqslant\left\|P_{U} J^{\prime}\left(u_{k}\right)\right\|^{2} /(2 L) .
$$

Assume that $\alpha_{k}^{\prime} \leqslant \gamma$, then $\alpha_{k}=\alpha_{k}^{\prime}$ and thus, as $\alpha=1 / L$, the inequalities

$$
J\left(u_{k}\right)-J\left(u_{k+1}\right) \geqslant J\left(u_{k}\right)-J\left(u_{k+1}^{\alpha}\right) \geqslant\left\|P_{U} J^{\prime}\left(u_{k}\right)\right\|^{2} /(2 L)
$$

hold true.

Assume that $\alpha_{k}^{\prime}>\gamma$, then $\alpha_{k}=\gamma$. We consider two cases: $\gamma \geqslant 1 / L$ and $\gamma<1 / L$. Since function $f_{k}(\alpha)$ decreases on the interval $\left(0, \alpha_{k}^{\prime}\right)$, in the first case we again obtain inequalities (44). In the second case $(\gamma<1 / L)$

$$
\gamma(1-\gamma L / 2) \geqslant \gamma / 2
$$

Thus, taking into consideration inequalities (42), (44), in each case we obtain the estimate

$$
J\left(u_{k}\right)-J\left(u_{k+1}\right) \geqslant c\left\|P_{U} J^{\prime}\left(u_{k}\right)\right\|^{2},
$$

where $c=\min [\gamma / 2,1 /(2 L)]$. It follows from the last estimate that sequence $\left\{J\left(u_{k}\right)\right\}_{k=0}^{\infty}$ decreases monotonically, the series $\sum_{k=0}^{\infty}\left\|P_{U} J^{\prime}\left(u_{k}\right)\right\|^{2}$ converges and the sought estimate

$$
\sum_{j=k}^{\infty}\left\|P_{U} J^{\prime}\left(u_{k}\right)\right\|^{2} \leqslant c^{-1}\left(J\left(u_{k}\right)-J *\right)
$$

holds true. Hence, condition (39) holds true and the proof is complete.

\section{Iteration Processes. Convergence TheOrem}

In view of Theorem 2 , in what follows we shall discuss only Problems $l=1,2$. Let us show that functionals $J_{l}(u)$ satisfy all the assumptions of Theorem 5 .

It is easy to make sure that functionals $J_{l}$ are convex. Indeed, for all $\alpha \in[0,1]$ and $u, v \in U_{l}$,

$$
\begin{aligned}
& J_{l}(\alpha u+(1-\alpha) v)=\|\alpha \operatorname{div} \mathbf{v}(u)+(1-\alpha) \operatorname{div} \mathbf{v}(v)\|_{L_{2}(\Omega)}^{2} \\
& \quad=\alpha^{2}\|\operatorname{div} \mathbf{v}(u)\|_{L_{2}(\Omega)}^{2}+(1-\alpha)^{2}\|\operatorname{div} \mathbf{v}(v)\|_{L_{2}(\Omega)}^{2}+2 \alpha(1-\alpha)(\operatorname{div} \mathbf{v}(u), \operatorname{div} \mathbf{v}(v))_{L_{2}(\Omega)} \\
& \quad=\alpha\|\operatorname{div} \mathbf{v}(u)\|_{L_{2}(\Omega)}^{2}+(1-\alpha)\|\operatorname{div} \mathbf{v}(v)\|_{L_{2}(\Omega)}^{2}-\alpha(1-\alpha)\|\operatorname{div} \mathbf{v}(u)-\operatorname{div} \mathbf{v}(v)\|_{L_{2}(\Omega)}^{2} \\
& \quad \leqslant \alpha J_{l}(u)+(1-\alpha) J_{l}(v) .
\end{aligned}
$$

The belonging of functionals $J_{l}$ to class $C^{1,1}(U)$ is implied by the results of Sections 2, 3, $J_{l, *}=\inf _{U} J_{l}(u)=0>-\infty$, sets $U_{l, *}=\left\{u \in U_{l}: J_{l}(u)=J_{l, *}\right\}$ consist of one point $u_{*}=p$, where $p$ is the sought pressure.

Thus, it follows from Theorem 5 that sequence $\left\{u_{k}\right\}_{k=0}^{\infty}$ defined by relations (6), (31), (33) converges weakly in corresponding spaces $H^{1}(\Omega)$ and $L_{2}(\Omega)$ for each initial approximations as $l=1,2$.

Theorem 6. Let $\boldsymbol{f} \in \boldsymbol{L}_{2}(\Omega), S \in C^{2}$. Then sequence $\left\{u_{k}\right\}_{k=0}^{\infty}$ defined by formulae (6), (33) as $l=1,2$ converge to $u_{*}=p$ weakly in $H^{1}(\Omega)$ and strongly in $L_{2}(\Omega)$ as $l=1$ for each initial approximation. As $l=1,2$, sequences $\left\{\boldsymbol{v}\left(u_{k}\right)\right\}_{k=0}^{\infty}$ converge to $\boldsymbol{v}$ strongly in $\boldsymbol{H}_{0}^{1}(\Omega)$, where $p$ and $\boldsymbol{v}$ is the solution to problem (1)-(3). 
Proof. As it was shown, the first statement is implied immediately by Theorem 5 . The validity of the second statement is implied by the following arguments. It is known (see, for instance, [1. Ch. 1, Sect. 1, Lm. 8]) that if domain $\Omega$ is bounded and its boundary is piece-wise smooth, then $H^{1}(\Omega)$ is compactly embedded into $L_{2}(\Omega)$ (i.e., a bounded in $H^{1}(\Omega)$ set is compact in $\left.L_{2}(\Omega)\right)$. The compactness and weak convergence in $\boldsymbol{H}^{1}(\Omega)$ of sequence $\left\{\mathbf{v}\left(u_{k}\right)\right\}_{k=0}^{\infty}$ implies its strong convergence in $\boldsymbol{L}_{2}(\Omega)$.

We then observe that the difference $\mathbf{v}_{k}-\mathbf{v}$, where $\mathbf{v}_{k}=\mathbf{v}\left(u_{k}\right)$, and $\mathbf{v}$ is the solution to problem (1)-(3), solves the problem

$$
-\nu \Delta\left(\mathbf{v}_{k}-\mathbf{v}\right)=-\nabla\left(u_{k}-p\right),\left.\left(\mathbf{v}_{k}-\mathbf{v}\right)\right|_{S}=0
$$

Multiplying the last equation by $\mathbf{v}_{k}-\mathbf{v}$ and integrating by parts, we obtain

$$
\left\|\nabla\left(\mathbf{v}_{k}-\mathbf{v}\right)\right\|_{L_{2}(\Omega)}=-\nu^{-1}\left(\nabla\left(u_{k}-p\right), \mathbf{v}_{k}-\mathbf{v}\right)_{L_{2}(\Omega)}=\nu^{-1}\left(u_{k}-p, \operatorname{div} \mathbf{v}_{k}\right)_{L_{2}(\Omega)} .
$$

Sequence $\left\{u_{k}\right\}_{k=0}^{\infty}$ is bounded in $L_{2}(\Omega)$ and it minimizes functional $J_{l}(u)$. Hence, $\operatorname{div} \mathbf{v}_{k} \rightarrow 0$ as $k \rightarrow \infty$. It follows that $\left(u_{k}-p \text {, } \operatorname{div} \mathbf{v}_{k}\right)_{L_{2}(\Omega)} \rightarrow 0$ as $k \rightarrow \infty$. Therefore, by relations 46$)$ we arrive at the last statement of the theorem.

The proven theorem allows us to formulate the iteration processes in differential form for solving problem (1)-(3).

The algorithm for $J_{1}(u)$

1. Choose initial $u_{0} \in U_{1}$, for instance, $u_{0}=0$.

2. Find velocity $\mathbf{v}_{k}\left(u_{k}\right)$ as the solution to vector Dirichlet problem (5).

3. Find the dual state $\mathbf{w}_{k}\left(u_{k}\right)$ by the solution to vector Dirichlet problem (10).

4. Determine $d_{k}=P_{U_{1}}\left(\rho\left(\mathbf{w}_{k}\right)\right)$, where $\rho\left(\mathbf{w}_{k}\right)$ is the solution to scalar Neumann problem (25).

5. Determine $\stackrel{\circ}{\mathbf{v}}_{k}\left(d_{k}\right)$ by the solution to vector Dirichlet problem (5) as $\mathbf{f}=0$.

6. Calculate $\alpha_{k}$ by formulae (31), (33) for found $\mathbf{v}_{k}\left(u_{k}\right), \stackrel{\circ}{\mathbf{v}}_{k}\left(d_{k}\right)$.

7. Recalculate control $u_{k+1}$ by formula (32) for found $\alpha_{k}, d_{k}$ and pass to step 2.

The algorithm for $J_{2}(u)$ is identical to the algorithm for $J_{1}(u)$ except step 4 :

$$
d_{k}=P_{U_{2}}\left(-\operatorname{div} \mathbf{w}_{k}\left(u_{k}\right)\right)
$$

The projection operators for both algorithms are calculated by the formula

$$
P_{U_{l}}(u)=u-\frac{(u, 1)_{L_{2}(\Omega)}}{(1,1)_{L_{2}(\Omega)}} \forall u \in U_{l}, l=1,2 .
$$

\section{Combined GRadient Method}

The matter is that we make in turn several steps of the first gradient method (i.e., $J^{\prime}(u)=$ $J_{1}^{\prime}(u)$ ), and then several steps of the second method (i.e., $J^{\prime}(u)=J_{2}^{\prime}(u)$ ). The following calculations show that combination of the methods ensures an acceleration of the convergence. This fact has a simple explanation. Functionals $J_{1}(u), J_{2}(u)$ attain the minimum via vanishing at point $u_{*}=p$ if and only if the dual state satisfies $\mathbf{w}\left(u_{*}\right)=0$. Indeed, if $\mathbf{w}\left(u_{k}\right) \neq 0$, then in the expansion of $\mathbf{w}\left(u_{k}\right)$ into the gradient and solenoidal parts at least one of the parts is non-zero. For example, let gradient part $\mathbf{w}\left(u_{k}\right)$ is non-zero, then the descent by gradient $J_{1}^{\prime}\left(u_{k}\right)$ ensures the lessening of the functional. The combined method consists in consecutive passing from using one gradient to using the other as soon as the quantity $\left\|J_{l}^{\prime}\left(u_{k}\right)\right\|,(l=1,2)$, becomes less than a prescribed quantity.

We note that the domains of functionals $U_{l},(l=1,2)$, are different and this is why one should make sure that finishing using one method of gradient descent we obtain a control $u_{k}$ belonging to the domain of the other functional. This condition is satisfied if the initial approximation satisfies $u_{0} \in U_{1}$. In order to make sure, it is sufficient to show that if $u_{0} \in H^{1}(\Omega)$, then $J_{2}^{\prime}\left(u_{0}\right) \in \boldsymbol{H}^{1}(\Omega)$. Indeed, if $u_{0} \in H^{1}(\Omega)$, then under the smoothness condition $S \in C^{2}$, 
the right hand in equation (5) satisfies $\mathbf{f}-\nabla u_{0} \in \boldsymbol{L}_{2}(\Omega)$ and hence for its solution we have $\mathbf{v}\left(u_{0}\right) \in \boldsymbol{H}^{2}(\Omega)$. It follows that the right hand side of equation $(10)$ belongs to $\boldsymbol{L}_{2}(\Omega)$ and hence, $\mathbf{w}\left(u_{0}\right) \in \boldsymbol{H}^{2}(\Omega)$. Therefore, $J_{2}^{\prime}\left(u_{0}\right)=-\operatorname{div} \mathbf{w}\left(u_{0}\right) \in \boldsymbol{H}^{1}(\Omega)$. Thus, all the terms of sequence $u_{k}$ obtained by the combined method belong to $U_{2}$.

If the application of gradient $J_{2}^{\prime}(u)$ ends up starting from some step of the iteration process, then in accordance with Theorem 6 , the iteration process converges weakly in $\boldsymbol{H}^{1}(\Omega)$ and strongly in $\boldsymbol{L}_{2}(\Omega)$. Otherwise one can prove the weak convergence of the iteration process in $\boldsymbol{L}_{2}(\Omega)$. The sequences of velocity vectors $\left\{\mathbf{v}\left(u_{k}\right)\right\}_{k=0}^{\infty}$ converge strongly in space $H_{0}^{1}(\Omega)$ by Theorem 6 .

\section{ON THE PROBLEM WITH INHOMOGENEOUS BOUNDARY CONDITION}

We provide some remarks on possible weakening of the conditions for the boundary and on application of the developed methods to solving Stokes problem with inhomogeneous boundary conditions.

The smoothness condition for the boundary of the domain $S \in C^{2}$ is employed only in using second energy inequality (12). However, as it was mentioned in [8], the condition of the boundary can be essentially weakened and for instance, any polyhedron or an arbitrary convex domain satisfy these weakened conditions.

To the solve Stokes problem with inhomogeneous boundary conditions

$$
\begin{aligned}
& -\nu \Delta \mathbf{v}=\mathbf{f}-\nabla p,\left.\mathbf{v}\right|_{S}=\vec{\varphi}, \\
& \operatorname{div} \mathbf{v}=0 \\
& (p, 1)_{L_{2}(\Omega)}=0
\end{aligned}
$$

we can apply the proposed method. At that, the difference to the case of homogeneous boundary conditions is that at each step of the descent by the gradient, one has to solve an inhomogeneous boundary condition (48) in order to determined velocity vector $\mathbf{v}(u)$ associated with control $u$. If control $u$ is increased by an increment $h$, then the increment of the velocity vector $\mathbf{v}(\nabla h)=\mathbf{v}(\nabla(u+h))-\mathbf{v}(\nabla u)$ is determined by the solution to the homogeneous boundary value problem as in the above considered cases. Now it is easy to see that the arguments used in the proof of Theorems 1, 2, 3 remain unchanged.

Thus, functionals $J_{l},(l=1,2)$, considered in spaces $H^{1}(\Omega)$ and $L_{2}(\Omega)$ are differentiable and satisfy Lipschitz condition in the case of inhomogeneous boundary conditions, too. Moreover, formula (31) determining parameters $\alpha_{k}$ remain true.

To justify the convergence of sequence $\left\{u_{k}\right\}_{k=0}^{\infty}$ constructed by the above proposed methods, we need to show that the sets of the minimum points for functionals $J_{l}$ is non-empty and bounded. Here it is sufficient to make sure that problem $(48)-(50)$ is solvable.

The solvability of problem (48)-(50) in class $\mathbf{v} \in \boldsymbol{H}^{2}(\Omega), \nabla p \in \boldsymbol{L}_{2}(\Omega)$ is guaranteed by [1, Ch. III, Sect. 5, Thm. 3] under the condition that domain $\Omega$ is bounded $\mathbf{f} \in \boldsymbol{L}_{2}(\Omega), S \in C^{2}$, $\left.\vec{\varphi}\right|_{S} \in H^{1+\frac{1}{2}},(\vec{\varphi}, \vec{n})_{L_{2}(S)}=0$. Therefore, under these conditions Theorem 6 holds true.

\section{Finite-DifFERENCE APPROXIMATION}

To construct grid analogues of differential iteration processes in Section 5, it is insufficient to employ arbitrary approximations for operators div and $\nabla$ and to guarantee the convergence. The authors faced this fact in numerical modelling. However, if we first approximate original problem (1)-(3) in finite-dimensional Sobolev spaces and construct the gradient method for this problem similarly to the above considered differential case, we succeed to obtain a converging iteration process, for which Theorem 6 holds true.

As in work [11, we consider a two-dimensional rectangular domain $\Omega_{h}=\left(0, l_{1}\right) \times\left(0, l_{2}\right)$ and a node uniformly non-staggered grid $\omega_{h}=\left\{x_{i, j}=\left(i h_{1}, j h_{2}\right), i=1, \ldots, N_{1}, j=1, \ldots, N_{2}\right\}$ with a 
boundary $S_{h}$ consisting of the boundary nodes except the corner points. Here $h_{\alpha}=l_{\alpha} /\left(N_{\alpha}+1\right)$ is the uniform step, and $N_{\alpha}$ is the amount of internal nodes of the set in the direction $\alpha=1,2$. In what follows we restrict ourselves by the case of the uniform grid $N_{1}=N_{2}=N$ in the square $l_{1}=l_{2}=l,\left(h_{1}=h_{2}=h\right)$.

We introduce a scalar product in a finite-dimensional space $L_{2}\left(\Omega_{h}\right)$ :

$$
\begin{aligned}
(y, z)_{L_{2}\left(\Omega_{h}\right)}= & h^{2} \sum_{x_{i, j} \in \Omega_{h}} y_{i, j} z_{i, j}+\frac{h^{2}}{2} \sum_{x_{i, j} \in S_{h}} y_{i, j} z_{i, j}=h^{2} \sum_{i=1}^{N} \sum_{j=1}^{N} y_{i, j} z_{i, j} \\
& +\frac{h^{2}}{2}\left(\sum_{i=1}^{N} y_{i, 0} z_{i, 0}+\sum_{i=1}^{N} y_{i, N+1} z_{i, N+1}+\sum_{j=1}^{N} y_{0, j} z_{0, j}+\sum_{j=1}^{N} y_{N+1, j} z_{N+1, j}\right),
\end{aligned}
$$

where $y_{i, j}=y\left(x_{i, j}\right), z_{i, j}=z\left(x_{i, j}\right)$.

By a consistent approximation of operators $\operatorname{div}_{h}$ and $\nabla_{h}$ we mean the preserving of the differential property of integration by parts:

$$
\left(\nabla_{h} p, \mathbf{v}\right)_{L_{2}\left(\Omega_{h}\right)}=-\left(p, \operatorname{div}_{h} \mathbf{v}\right)_{L_{2}\left(\Omega_{h}\right)} \forall \mathbf{v} \in H_{0}^{1}\left(\Omega_{h}\right)
$$

Relation (51) is satisfied by first order oriented differences [11]:

$$
\begin{aligned}
\operatorname{div}_{h} \mathbf{v}_{i, j} & =\frac{v_{i, j}^{(1)}-v_{i-1, j}^{(1)}}{h}+\frac{v_{i, j}^{(2)}-v_{i, j-1}^{(2)}}{h}, i=1 \ldots N+1, j=1 \ldots N+1, \\
\nabla_{h} p_{i, j} & =\left(\frac{p_{i+1, j}-p_{i, j}}{h}, \frac{p_{i, j+1}-p_{i, j}}{h}\right), i=0 \ldots N, j=0 \ldots N
\end{aligned}
$$

It is obvious that in this case operator $-\Delta_{h}=-\operatorname{div}_{h} \nabla_{h}$ is the standard five-points difference pattern for the Dirichlet problem [12].

Hence, the grid analogue of problem (1)-(3) is the problem

$$
\begin{aligned}
& -\nu \Delta_{h} \mathbf{v}=\mathbf{f}_{h}-\nabla_{h} p,\left.\mathbf{v}\right|_{S_{h}}=0 \\
& \operatorname{div}_{h} \mathbf{v}=0 \\
& \quad(p, 1)_{L_{2}\left(\Omega_{h}\right)}=0
\end{aligned}
$$

for which we write the iteration processes from Section 5 in a form convenient for a computer realization. We shall consider $J_{0}(\vec{u})$ instead of $J_{1}(u)$.

The algorithm for $J_{0}(\vec{u})$

$$
\begin{aligned}
& -\nu \Delta_{h} \mathbf{v}_{k}=\mathbf{f}_{h}-\vec{u}_{k},\left.\mathbf{v}_{k}\right|_{S_{h}}=0 ; \\
& -\nu \Delta_{h} \mathbf{w}_{k}=\nabla_{h} \operatorname{div}_{h} \mathbf{v}_{k},\left.\mathbf{w}_{k}\right|_{S_{h}}=0 ; \\
& -\Delta \rho_{k}=-\operatorname{div}_{h} \mathbf{w}_{k},\left.\frac{\partial \rho_{k}}{\partial \vec{n}}\right|_{S_{h}}=\left.\left(\mathbf{w}_{k} \cdot \vec{n}\right)\right|_{S_{h}} ; \\
& d_{k}=P_{U_{1}}\left(\rho_{k}\right) ; \\
& -\nu \Delta_{h} \stackrel{\circ}{\mathbf{v}}_{k}=-\nabla_{h} d_{k},\left.\stackrel{\circ}{\mathbf{v}}_{k}\right|_{S_{h}}=0 ; \\
& \alpha_{k}=\min \left[\alpha_{k}^{\prime}, \gamma\right], \alpha_{k}^{\prime}=\frac{\left(\operatorname{div}_{h} \mathbf{v}_{k}, \operatorname{div}_{h} \stackrel{\circ}{\mathbf{v}}_{k}\right)_{L_{2}\left(\Omega_{h}\right)}}{\left\|\operatorname{div}_{h} \stackrel{\circ}{\mathbf{v}}_{k}\right\|_{L_{2}\left(\Omega_{h}\right)}^{2}} ; \\
& \vec{u}_{k+1}=\vec{u}_{k}-\alpha_{k} \nabla_{h} d_{k} .
\end{aligned}
$$


The algorithm for $J_{2}(u)$

$$
\begin{aligned}
& -\nu \Delta_{h} \mathbf{v}_{k}=\mathbf{f}_{h}-\nabla u_{k},\left.\mathbf{v}_{k}\right|_{S_{h}}=0 \\
& -\nu \Delta_{h} \mathbf{w}_{k}=\nabla_{h} \operatorname{div}_{h} \mathbf{v}_{k},\left.\mathbf{w}_{k}\right|_{S_{h}}=0 \\
& \rho_{k}=-\operatorname{div}_{h} \mathbf{w}_{k} \\
& d_{k}=P_{U_{1}}\left(\rho_{k}\right) ; \\
& -\nu \Delta_{h} \stackrel{\circ}{\mathbf{v}}_{k}=-\nabla_{h} d_{k},\left.\stackrel{\circ}{\mathbf{v}}_{k}\right|_{S_{h}}=0 \\
& \alpha_{k}=\min \left[\alpha_{k}^{\prime}, \gamma\right] \\
& \alpha_{k}^{\prime}=\frac{\left(\operatorname{div}_{h} \mathbf{v}_{k}, \operatorname{div}_{h} \stackrel{\circ}{\mathbf{v}}_{k}\right)_{L_{2}\left(\Omega_{h}\right)}}{\left\|\operatorname{div}_{h} \stackrel{\circ}{k}_{k}\right\|_{L_{2}\left(\Omega_{h}\right)}^{2}} \\
& u_{k+1}=u_{k}-\alpha_{k} d_{k}
\end{aligned}
$$

For both algorithms

$$
P_{U_{l}}\left(\rho_{k}\right)=\rho_{k}-\frac{\left(\rho_{k}, 1\right)_{L_{2}\left(\Omega_{h}\right)}}{(1,1)_{L_{2}\left(\Omega_{h}\right)}},
$$

where, as it is easy to make sure, $(1,1)_{L_{2}\left(\Omega_{h}\right)}=l_{1} l_{2}$.

\section{Solution to Degenerate Neumann PROBlem}

It is well known that the solvability condition of Neumann differential problem

$$
-\Delta \rho=f,\left.\frac{\partial \rho}{\partial \vec{n}}\right|_{S}=g
$$

is the identity

$$
(f, 1)_{L_{2}(\Omega)}+(g, 1)_{L_{2}(S)}=0
$$

which for problem (24) becomes Gauss-Ostrogradsky formula

$$
\left(\operatorname{div} \mathbf{w}_{k}, 1\right)_{L_{2}(\Omega)}=\left(\left(\mathbf{w}_{k} \cdot \vec{n}\right), 1\right)_{L_{2}(S)} .
$$

The validity of the last formula for the grid case can be checked straightforwardly if scalar product in $L_{2}\left(S_{h}\right)$ is introduced as

$$
\begin{aligned}
(g, 1)_{L_{2}\left(S_{h}\right)}= & h \sum_{i=1}^{N}\left(g_{0, i}+g_{2, i}\right)+h \sum_{j=1}^{N}\left(g_{1, j}+g_{3, j}\right) \\
& +\frac{h}{2}\left(g_{0,0}+g_{1,0}+g_{2, N+1}+g_{3, N+1}+g_{2,0}+g_{1, N+1}+g_{0, N+1}+g_{3,0}\right) \forall g \in L_{2}\left(\Omega_{h}\right) .
\end{aligned}
$$

We introduce the notation for the components of vector $\mathbf{w}_{k}=\left(w_{k}^{(1)}, w_{k}^{(2)}\right)$. By the definition, $\partial \rho_{k} /\left.\partial \vec{n}\right|_{S_{h}}=\left.\left(\nabla_{h} \rho_{k} \cdot \vec{n}\right)\right|_{S_{h}}$ and this is why the boundary condition for problem (55) is determined by the identity $\left.\left(\nabla_{h} \rho_{k}-\mathbf{w}_{k}\right)\right|_{S_{h}}=0$ :

$$
\begin{aligned}
& \frac{\left(\rho_{k}\right)_{1, j}-\left(\rho_{k}\right)_{0, j}}{h}=\left(w_{k}^{(1)}\right)_{0, j}, \frac{\left(\rho_{k}\right)_{N+2, j}-\left(\rho_{k}\right)_{N+1, j}}{h}=\left(w_{k}^{(1)}\right)_{N+1, j}, j \in \overline{1, N} \\
& \frac{\left(\rho_{k}\right)_{i, 1}-\left(\rho_{k}\right)_{i, 0}}{h}=\left(w_{k}^{(2)}\right)_{i, 0}, \frac{\left(\rho_{k}\right)_{i, N+2}-\left(\rho_{k}\right)_{i, N+1}}{h}=\left(w_{k}^{(2)}\right)_{i, N+1}, i \in \overline{1, N} .
\end{aligned}
$$

By these formulae we see that we need to introduce fictitious layers of point in each of the directions with indices $N+2$ beyond grid domain $\omega_{h} \cup S_{h}$. Then we write a $(N+2) \times(N+2)$ system of linear algebraic equations for solution of problem (55):

$$
A z=b,
$$

where $z_{C E L L(i, j)}=\left(\rho_{k}\right)_{i, j}$, while operator $C E L L(i, j)=i(N+2)+j(i, j \in \overline{0, N+1})$ defines line-by-line format for storage of a two-dimensional vector in one-dimensional array. 
All the elements of the row row $=C E L L(i, j)(i, j \in \overline{0, N+1})$ of matrix $A$ vanish except

$$
\begin{aligned}
& A_{\text {row }, C E L L(i, j)}=\left\{\begin{array}{l}
4, i, j \in \overline{1, N} \\
3, i=0 \text { and } j \in \overline{1, N} \text { or } i=N+1 \text { and } j \in \overline{1, N} \\
3, j=0 \text { and } i \in \overline{1, N} \text { or } j=N+1 \text { and } i \in \overline{1, N} \\
2, i=0 \text { and } j=0 \text { or } i=0 \text { and } j=N+1 ; \\
2, i=N+1 \text { and } j=0 \text { or } i=N+1 \text { and } j=N+1 .
\end{array}\right. \\
& A_{\text {row, } C E L L(i-1, j)}=-1, i \geqslant 1 ; \\
& A_{\text {row }, C E L L(i+1, j)}=-1, i \leqslant N ; \\
& A_{\text {row }, C E L L(i, j-1)}=-1, j \geqslant 1 ; \\
& A_{\text {row }, C E L L(i, j+1)}=-1, j \leqslant N .
\end{aligned}
$$

The vector in the right hand side can be calculated by the formulae

$$
b_{\text {row }}=-h^{2} \operatorname{div}_{h}\left(\mathbf{w}_{k}\right)_{i, j}+h\left\{\begin{array}{l}
0, i \in \overline{1, N}, j \in \overline{1, N} \\
\left(w_{k}^{(1)}\right)_{0, j}, i=1, j \in \overline{0, N+1} \\
\left(w_{k}^{(2)}\right)_{i, N+1}, j=N+1, i \in \overline{0, N+1} \\
\left(w_{k}^{(1)}\right)_{N+1, j}, i=N+1, j \in \overline{0, N+1} \\
\left(w_{k}^{(2)}\right)_{i, 0}, j=1, i \in \overline{0, N+1 .}
\end{array}\right.
$$

System (57) has a dispersive structure and it can be effectively solved by an appropriate iteration method with a preconditioning.

\section{NUMERICAL EXPERIMENTS}

Traditionally, the primary verification of numerical methods is made for the problems with a known analytic solution. For problem (1)-(3) it means that defining solenoidal $\mathbf{v}^{*}$ and $p^{*}$ in the condition $\left(p^{*}, 1\right)_{L_{2}(\Omega)}$, we can calculate the grid right hand side $\mathbf{f}_{h}=\left(-\nu \Delta \mathbf{v}^{*}+\nabla p^{*}\right)_{h}$ and to find vectors $\mathbf{v}_{k}, u_{k}$ as the convergence results of the algorithms in Section 9 ,

We consider a test example in work [4]. In domain $\Omega=[-\pi / 2,3 \pi / 2] \times[-\pi, \pi]$ we solve Stokes problem (1)-(3), where for $\nu=1$ we define a solenoidal analytic solution $\mathbf{v}^{*}(x, y)=$ $\{(1+\sin x) \sin y, \cos x(1+\cos y)\}, p^{*}(x, y)=\sin x \cos (2 y)$, for which $\left.\mathbf{v}^{*}\right|_{S}=0$.

Problem (52)-(54) was solved on the sequence of grids $31 \times 31,63 \times 63,127 \times 127,255 \times 255$ by means of algorithms $J_{2}(u), J_{0}(\vec{u})$, as well as by their combination. Dirichlet problems for Poisson equation were solved by the fast Fourier transformation method 12 that explains the choice of the amount of the internal nodes in the grids by the rule $N_{1}=N_{2}=N=2^{m}-1,(m=5,6,7,8)$. Neumann problem (57) was solved iteratively by the conjugate gradient method with a diagonal preconditioning. In all the algorithms all the iterations were made until $\left\|p_{k}-p_{k-1}\right\|_{C\left(\Omega_{h}\right)} \geqslant 10^{-6}$ or $\left\|\operatorname{div}_{h} \mathbf{v}_{k}\right\|_{C\left(\Omega_{h}\right)} \geqslant 10^{-6}$, where $\|\cdot\|_{C\left(\Omega_{h}\right)}$ is the uniform grid norm, that is, the maximum among all the absolute values of a function on grid $\omega_{h}$. As the initial approximation vector we used $u_{0}=0$.

The results of the calculations by means of the program developed by the authors in language $\mathrm{C}++$ are given in Tables 1, 2. In Figures 1, 2 we present the decay dynamics of the norm of the divergence of velocity vector.

The calculations by algorithm $J_{0}(\vec{u})$ are not presented because of the found numerical instability of the calculations.

The influence of parameter $\gamma$ on the convergence of the modified gradient descent method with finding parameter $\alpha_{k}$ by formulae (33), (31) is demonstrated in Figure 2. As the basis, we choose a combined method, where after one step $J_{0}(\vec{u})$, the following iterations were made $\mathrm{y}$ 


\begin{tabular}{l|l|l|l|l}
\hline Grid & Iterations & $\left\|\mathbf{v}_{k}-\mathbf{v}^{*}\right\|_{C\left(\Omega_{h}\right)}$ & $\left\|p_{k}-p^{*}\right\|_{C\left(\Omega_{h}\right)}$ & Error $\left\|\mathbf{f}_{h}-\nabla_{h} p_{k}+\Delta_{h} \mathbf{v}_{k}\right\|_{C\left(\Omega_{h}\right)}$ \\
\hline $31 \times 31$ & 200 & $1.92952 \mathrm{e}-11$ & $3.05475 \mathrm{e}-5$ & $1.54609 \mathrm{e}-9$ \\
$63 \times 63$ & 242 & $7.96903 \mathrm{e}-12$ & $3.81565 \mathrm{e}-6$ & $2.72057 \mathrm{e}-9$ \\
$127 \times 127$ & 262 & $3.3259 \mathrm{e}-12$ & $4.76882 \mathrm{e}-7$ & $5.99933 \mathrm{e}-9$ \\
$255 \times 255$ & 265 & $1.41589 \mathrm{e}-12$ & $5.96158 \mathrm{e}-8$ & $1.26728 \mathrm{e}-8$ \\
\hline
\end{tabular}

TABLE 1. The results of the calculations by algorithm $J_{2}(u)$

\begin{tabular}{l|l|l|l|l}
\hline Grid & Iterations & $\left\|\mathbf{v}_{k}-\mathbf{v}^{*}\right\|_{C\left(\Omega_{h}\right)}$ & $\left\|p_{k}-p^{*}\right\|_{C\left(\Omega_{h}\right)}$ & Error $\left\|\mathbf{f}_{h}-\nabla_{h} p_{k}+\Delta_{h} \mathbf{v}_{k}\right\|_{C\left(\Omega_{h}\right)}$ \\
\hline $31 \times 31$ & 167 & $1.80279 \mathrm{e}-11$ & $1.7414 \mathrm{e}-6$ & $1.15983 \mathrm{e}-9$ \\
$63 \times 63$ & 143 & $7.09932 \mathrm{e}-12$ & $1.56985 \mathrm{e}-5$ & $2.04383 \mathrm{e}-9$ \\
$127 \times 127$ & 163 & $4.07434 \mathrm{e}-12$ & $1.0086 \mathrm{e}-5$ & $5.90967 \mathrm{e}-9$ \\
$255 \times 255$ & 219 & $2.13486 \mathrm{e}-12$ & $5.4197 \mathrm{e}-6$ & $1.1914 \mathrm{e}-8$ \\
\hline
\end{tabular}

TABLE 2. The results of calculations by a combined algorithm, where after one step $J_{0}(\vec{u})$, the iterations follow algorithm $J_{2}(u)$

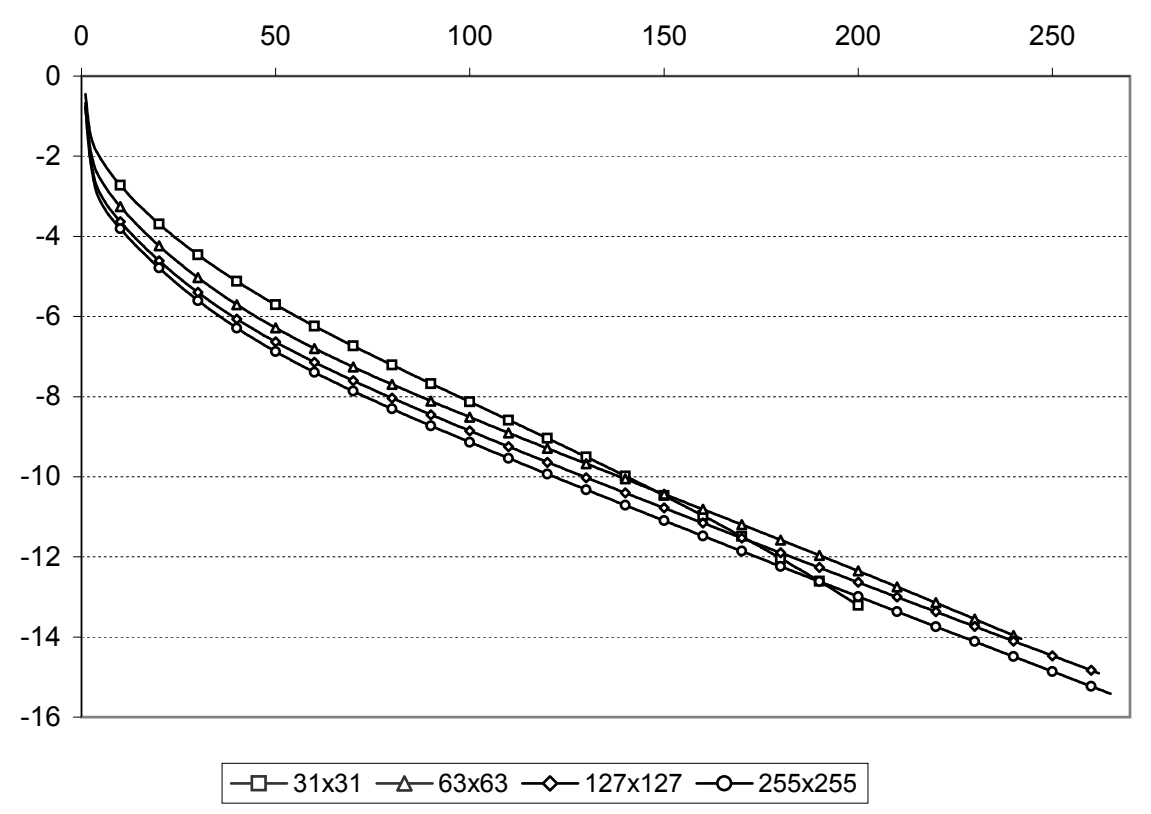

FiguRE 1. The graph of $\log _{10}\left(\operatorname{div}_{h} \mathbf{v}_{k}\right)$ for algorithm $J_{2}(u)$ depending on the iteration step

algorithm $J_{2}(u)$. The optimal value $\gamma$ for the considered grids is a number ranging between 9 and 11. This number is most likely to be in other ranges under other conditions of the problem. Comparing Table 3 and Table 2, we observe the reduction of the amount of iterations.

\begin{tabular}{l|l|l|l|l}
\hline Grid & Iterations & $\left\|\tilde{\mathbf{v}}_{k}-\mathbf{v}^{*}\right\|_{C\left(\Omega_{h}\right)}$ & $\left\|u_{k}-p^{*}\right\|_{C\left(\Omega_{h}\right)}$ & Error $\left\|\mathbf{f}-\nabla_{h} u_{k}+\Delta_{h} \tilde{\mathbf{v}}_{k}\right\|_{C\left(\Omega_{h}\right)}$ \\
\hline $31 \times 31$ & 113 & $2.53668 \mathrm{e}-12$ & $2.00158 \mathrm{e}-6$ & $1.56719 \mathrm{e}-9$ \\
$63 \times 63$ & 121 & $0.999382 \mathrm{e}-11$ & $1.00493 \mathrm{e}-5$ & $2.94942 \mathrm{e}-9$ \\
$127 \times 127$ & 133 & $0.497812 \mathrm{e}-11$ & $0.502867 \mathrm{e}-5$ & $4.47148 \mathrm{e}-9$ \\
$255 \times 255$ & 189 & $0.248806 \mathrm{e}-11$ & $0.251476 \mathrm{e}-6$ & $9.65243 \mathrm{e}-8$ \\
\hline
\end{tabular}

TABLE 3. Results of the calculation by the combined algorithm as $\gamma=10$ 


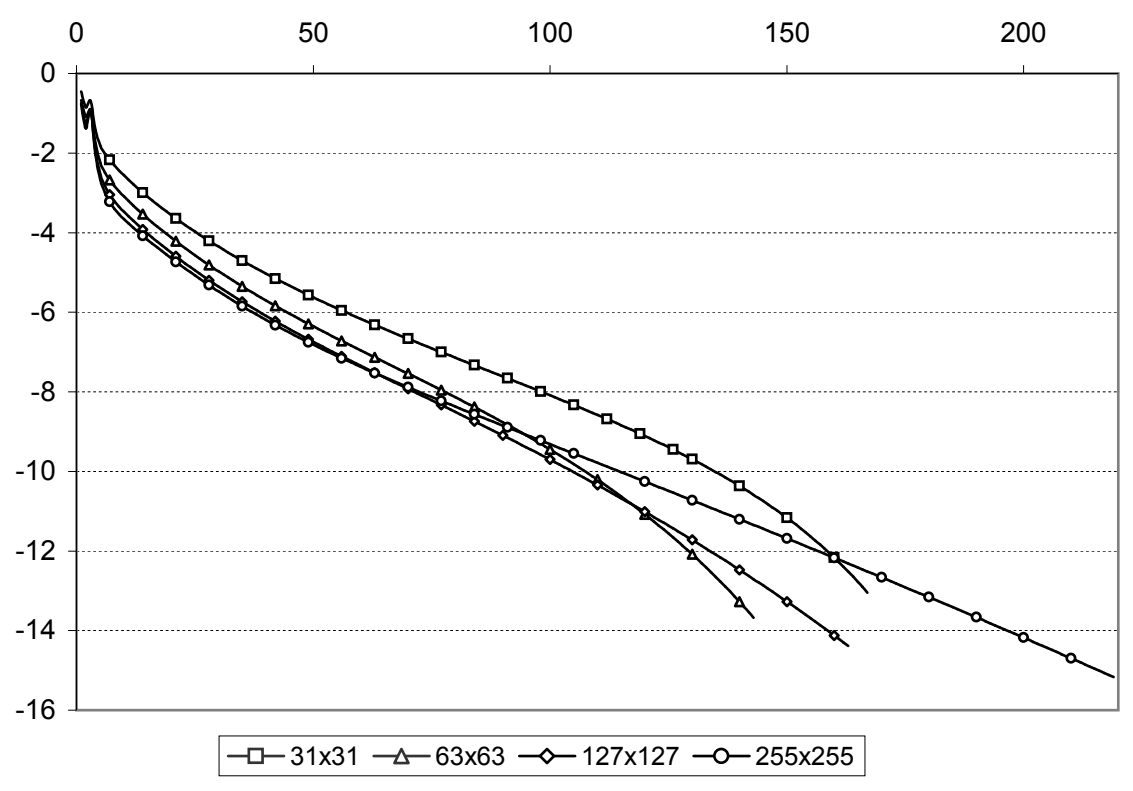

FIGURE 2. The graph $\log _{10}\left(\operatorname{div}_{h} \mathbf{v}_{k}\right)$ for the combined algorithm depending on the iteration step

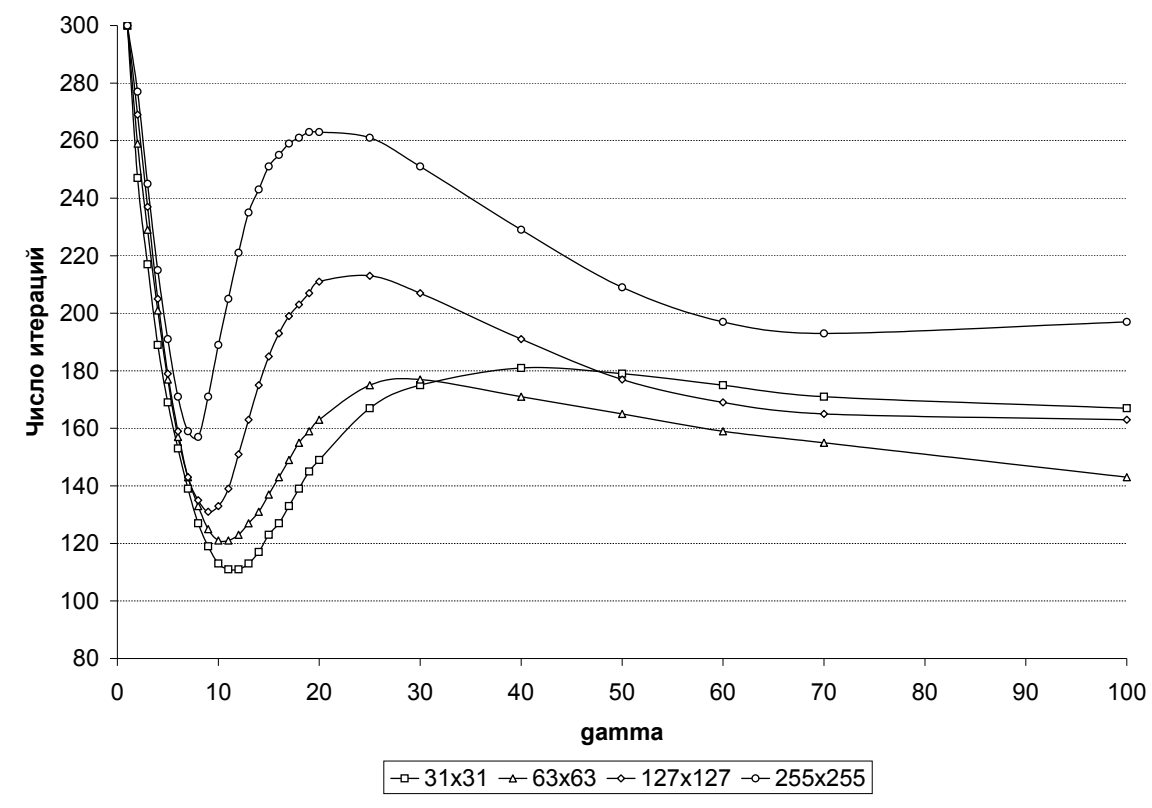

Figure 3. Dependence of the amount of iterations on $\gamma$ for the combined method

\section{Conclusion}

In conclusion we note that first, two constructed iteration process in Section 6 are written in differential form and invariant in the dimension of the considered Stokes problem $(n=2,3)$. Second, the reduction of the original problem to a series of much simpler Dirichlet and Neumann problem allows us to solve these problems by known effective grid methods.

In the present work we restrict ourselves by constructing only two-dimensional finitedifference schemes, for which we studied the efficiency of the proposed modified steepest descent method with parameter $\gamma$. 
Algorithm $J_{2}(u)$ is simple for computer realization but it converges slower than the combined method.

Comparison with numerical results in work [4] shows that even while applying the basic steepest descent method, the algorithms proposed in the present work require approximately twice less iterations for achieving the precision in velocity at the second position for the comparable grids.

\section{BIBLIOGRAPHY}

1. O.A. Ladyzhenskaya. Mathematical problems of the dynamics of a viscous incompressible fluids. Nauka, Moscow (1970). [Gordon and Breach, New York (1969).]

2. R. Temam. Navier-Stokes equations. Theory and numerical analysis. North Holland Publ. Co., Amsterdam (1979).

3. I. Ekeland, R. Temam. Convex analysis and variational problems. Stud. Math. Appl. 1. NorthHolland Publishing Company, Amsterdam (1976).

4. V.I. Agoshkov, C. Bardos, S.N. Buleev. Solution of the Stokes problem as an inverse problem // Comp. Meth. Appl. Math. 2:3, 213-232 (2002).

5. B.V. Pal'tsev, M.B. Solov'ev, I.I. Chechel'. On the development of iterative methods with boundary condition splitting for solving boundary and initial-boundary value problems for the linearized and nonlinear Navier-Stokes equations // Zhurn. Vychisl. Matem. Matem. Fiz. 51:1, 74-95 (2011). [Comp. Math. Math. Phys. 51:1, 68-87 (2011).]

6. F.P. Vasil'ev. Methods for solving extremal problems. Minimization problems in function spaces, regularization, approximation. Nauka, Moscow (1981).

7. F.P. Vasil'ev. Numerical methods for solving of extremal problems. Nauka, Moscow (1988).

8. O.A. Ladyzhenskaya. The boundary value problems of mathematical physics. Nauka, Moscow (1973). [Appl. Math. Sci. 49. Springer-Verlag, New York (1985).]

9. O.A. Ladyzhenskaya. Relationship between the Stokes problem and decompositions of the spaces $W_{2}^{1}$ and $W_{2}^{(-1)} / /$ Algeb. Anal. 13:4, 119-133 (2001). [St. Petersburg Math. J. 13:4, 601-612 (2002).]

10. Golichev I.I. Modified gradient fastest descent method for solving linearized non-stationary NavierStokes equations // Ufimskij Matem. Zhurn. 5:4, 60-76 (2013). [Ufa Math. J. 5:4, 58-74 (2013).]

11. A.G. Churbanov, A.N. Pavlov, P.N. Vabishchevich. Operator-splitting methods for the incompressible Navier-Stokes equations on non-staggered grids. Part 1: First-order schemes // Int. J. Numer. Meth. Fluids. 21:8, 617-640 (1995).

12. A.A. Samarskij, A.V. Gulin. Numerical methods. Nauka, Moscow (1989). (in Russian).

Golichev Iosif Iosifovich

Institute of Mathematics with Computer Center,

Ufa Scientific Center, RAS

Chenryshevsky str. 112,

450008, Ufa, Russia

Ufa Branch of Financial University

at the Goverment of Russian Federation

Revolution str. 169,

450005, Ufa, Russia

E-mail: Golichev_II@mail.ru

Sharipov Timur Rafailevich,

Scientific Production enterprise "ATP"

Kuznecovskii zaton 22, building 2,

450103, Ufa, Russia

E-mail: SharipovTR@gmail.com 
Luchnikova Natalia Iosifovna,

Ufa Branch of Financial University

at the Goverment of Russian Federation

Revolution str. 169,

450005, Ufa, Russia

E-mail: Luchnikova_NI_77@mail.ru 